\title{
Periastron shifts of stellar orbits near the Galactic Center
}

\author{
G. F. Rubilar ${ }^{1}$ and A. Eckart ${ }^{2}$ \\ 1 Institut für Theoretische Physik, Universität zu Köln, Zülpicherstraße 77, 50937 Köln, Germany \\ 2 I. Physikalisches Institut, Universität zu Köln, Zülpicherstraße 77, 50937 Köln, Germany \\ e-mail: eckart@ph1.uni-koeln.de
}

Received 22 December 2000 / Accepted 2 May 2001

\begin{abstract}
The presence of a $2.9 \pm 0.4$ million solar mass object in the central stellar cluster of the Milky Way has recently been demonstrated via measurements of the stellar proper motions and radial velocities. This mass is located at the position of the compact radio source Sagittarius $\mathrm{A}^{*}\left(\mathrm{Sgr} \mathrm{A}^{*}\right)$ at a distance of $R_{\mathrm{o}}=8.0 \mathrm{kpc}$ and is most likely present in the form of a massive black hole $(\mathrm{BH})$. Some of the stars have a projected distance to Sgr A* of $\leq 0.005 \mathrm{pc}$ and have proper motion velocities of up to $1400 \mathrm{~km} \mathrm{~s}^{-1}$. Recent measurements indicate that their orbits show significant curvatures indicating that the stars indeed orbit the central compact object. Detailed measurements of the stellar orbits close to Sgr A* will allow us to precisely determine the distribution of this mass. With an increased point source sensitivity due to the combination of large telescope apertures, adaptive optics, and - in the very near future - NIR interferometry it is likely that stars with orbital time scales of the order of one year will be detected. Theses sources, however, will most likely not be on simple Keplerian orbits. The effects of measurable prograde relativistic and retrograde Newtonian periastron shifts will result in rosetta shaped orbits. A substantial Newtonian periastron rotation can already be expected if only a few percent of the central mass are extended. We discuss the conditions under which an extended mass can (over-) compensate the relativistic periastron shift. We also demonstrate that measuring a single periastron shift is not sufficient to determine the distribution of an extended mass component. A periastron shift will allow us to determine the inclination of the stellar orbits and to derive inclination corrected shift values. These have to be acquired for three stars on orbits with different energy or angular momentum in order to unambiguously solve for the compactness, extent and shape of any extended mass contribution.
\end{abstract}

Key words. Galaxy: center - Galaxy: kinematics and dynamics - relativity - stars: kinematics

\section{Introduction}

Over the recent years high angular resolution HST and ground based observations of the velocity fields of stars and gas have provided convincing evidence that a central dark mass concentration is present in a large number of nearby external galactic nuclei (Kormendy \& Richstone 1995; Magorrian et al. 1998; Richstone et al. 1998). These dark central masses are most likely to be massive black holes. The two cases which are most convincing are the Galactic Center (Eckart \& Genzel 1996, 1997; Genzel et al. 1997, 2000; Ghez et al. 1998, 2000) and the nucleus of NGC 4258 (Greenhill et al. 1995; Myoshi et al. 1995). Theoretical estimates of the stability of these nuclear masses at their measured densities indicate that they cannot be stable for more than a few $10^{7}$ years before they collapse to a massive BH (Maoz 1998). With a distance of only $8 \mathrm{kpc}$, the Galactic Center is the closest nucleus. A first detection of a NIR counter part of the compact radio source Sgr A* at the center of the Milky Way has

Send offprint requests to: G. F. Rubilar, e-mail: gr@thp.uni-koeln.de been reported by Genzel et al. (1997). In Fig. 1 we show the enclosed mass distribution as a function of separation from the position of Sgr A* (black solid line) together with a summary of the current results from observations and mass modeling. With the exception of small amounts of anisotropy, the line-of-sight and proper motions velocity dispersions of these stars are in very good agreement and follow a Kepler law as expected for the presence of a central point mass (Sellgren et al. 1990; Krabbe et al. 1995; Haller et al. 1996; Eckart \& Genzel 1996, 1997; Genzel et al. 1996, 1997, 2000; Ghez et al. 1998, 2000). LeonardMerritt projected mass estimators (Leonard \& Merritt 1989) and Jeans equation modeling of the stellar velocity data result in a central mass of $(2.9 \pm 0.4) \times 10^{6} M_{\odot}$ with a mass-to-luminosity ratio of $M_{\mathrm{L}}>100 M_{\odot} / L_{\odot}($ Genzel et al. 2000). The current measurements indicate that if all the mass is concentrated in a single component its density has to be $>3.7 \times 10^{12} M_{\odot} \mathrm{pc}^{-3}$ (Genzel et al. 2000, 1997; Ghez et al. 2000, 1998). The recent measurements of orbital curvature for at least three sources (Ghez et al. 2000 ) indicate that the stars truly orbit a common central mass located at a position that agrees to within less than 
50 mas with that of Sgr A*. The orbit of each of these stars can be used to estimate the enclosed mass and mass density. For a general central matter distribution, the orbits in the central nuclear cluster will not be simple Keplerian ellipses. Non-closed orbits can be produced by relativistic effects and also by an extended mass distribution, and for most cases, except for some highly eccentric orbits, this second effect is expected to be the dominant one.

A first analysis of the expected periastron shifts has been given by Fragile \& Mathews (2000, see also Munyaneza et al. 1998; Jaroszyński 1998). The present paper is an extension of this analysis. In those cases in which we consider a central black hole we assume that it is non-rotating since Jaroszyński (1998) and Fragile \& Mathews (2000) have shown that the effects of the black holes angular momentum (as least on the currently observable stars) are probably negligible. We also assume that the stars within the central arcsecond are O-stars and that their orbits are not influenced significantly by tidal effects on their surfaces.

After briefly summarizing in Sect. 2 the effect of only a central BH, we concentrate in Sect. 3 on a post-Newtonian description of orbits in a potential due to a combination of a central $\mathrm{BH}$ and a variety of pressureless, static extended matter distributions. In Sect. 4 we then discuss observational constraints and show that the expected relativistic and Newtonian periastron shifts can indeed be measured with new instrumentation that is present or under construction.

\section{Compact mass distribution}

Typical orbital time scales that are within reach with current and near future observational techniques are listed in Table 1. Orbital time scales at angular resolutions of less than $\sim 20$ mas are less than one year. In order to detect any periastron shift it is therefore extremely desirable to find and track the motion of stars that are as close to the center as possible. The current estimate of the stellar surface density for stars brighter than $K=17$ is $\nu_{17} \approx 20 \operatorname{arcsec}^{-2}$ (Genzel et al. 1997; Alexander \& Sternberg 1999; Ghez et al. 1998). Jaroszyński (1999) derives a stellar surface density for stars brighter than $K=21$ of $\nu_{21} \approx 25 \times \nu_{17}$. The detection of stars close to $\mathrm{Sgr} \mathrm{A}^{*}$ with short orbital time scales (see Table 1) therefore appears to be entirely feasible, especially in the presence of a stellar cusp (Alexander 1999). Such cusps can be regarded as quasisteady state configurations (e.g. Murphy et al. 1991) and the actual time-averaged number of cusp stars at small separations from the very center is large.

First, we consider the case where all the mass in the central region is in form of a single $\mathrm{BH}$. In this case the relativistic prograde periastron advance per revolution is given by (see Weinberg 1972, Eq. (8.6.11))

$$
\Delta \varphi=\frac{6 \pi G}{c^{2}} \frac{M}{a\left(1-e^{2}\right)}
$$

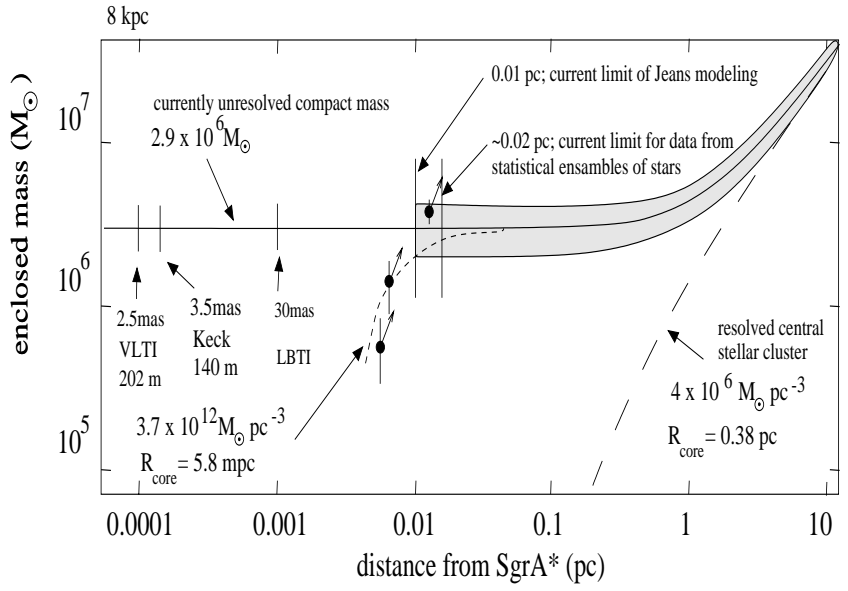

Fig. 1. A summary of mass modeling results in the central $10 \mathrm{pc}$ of the Galaxy from stellar and gas dynamics (Genzel et al. 2000, 1997, 1996; Ghez et al. 2000, 1998; Eckart \& Genzel $2000,1997)$. The long dashed curve represents the mass model for the stellar cluster $M_{\mathrm{L}}(2 \mu \mathrm{m})=2, r_{\text {core }}=0.38 \mathrm{pc}, \rho(r=$ $0)=3.5 \times 10^{6} M_{\odot} \mathrm{pc}^{-3}$. The continuous curve is the sum of this visible stellar cluster, plus a point mass of $2.9 \times 10^{6} M_{\odot}$. The short dashed curve is the sum of the visible stellar cluster, plus an $\alpha=5$ Plummer model (see Eq. (9)) of a dark cluster of central density $3.7 \times 10^{12} M_{\odot} \mathrm{pc}^{-3}$ and $r_{0}=0.0058 \mathrm{pc}$. The radii (as well as the corresponding angular resolutions and baselines for a wavelength of $2.2 \mu \mathrm{m}$ ) at which the enclosed mass can be investigated with NIR interferometers in the very near future are indicated (see also Sect. 4). The points with error bars and arrows indicate the lower limits on the enclosed mass derived from stellar accelerations (Ghez et al. 2000).

with $a$ being the semi-major axis and $e$ the eccentricity of the orbit, respectively (see also Appendix A). The positional shift of the apoastron

$\Delta s \approx a(1+e) \Delta \varphi=\frac{6 \pi G}{c^{2}} \frac{M}{1-e}$,

is, to first order, independent of the semi-major axis $a$ and only depends on the eccentricity $e$ of the orbit. Therefore, the accuracy with which positions can be measured impose a lower limit on the eccentricities of orbits for which relativistic periastron shifts can be observed. For an accuracy of 1 mas $=0.04 \mathrm{mpc}$ this corresponds to $e>0.93$ and for an accuracy of 0.1 mas $=0.004 \mathrm{mpc}, e>0.35$.

The gravitational length scale determined by the mass $M$ is

$\frac{G M}{c^{2}}=1.38 \times 10^{-4} \mathrm{mpc} \approx 2.76 \times 10^{-2} \mathrm{AU}$.

This equals half the Schwarzschild radius and determines the scale of distances at which the relativistic effects are dominant. In Fig. 2 we demonstrate the effect of the relativistic periastron shift for a star at the apoastron distance of 100 gravitational scale lengths. The prograde rosetta shaped trajectory has been calculated by solving Eq. (A.8); see also Sect. 3.2.

In order to appreciate the magnitude of the relativistic periastron shift for stellar orbits close to the Galactic 
Table 1. Angular and linear resolution versus orbital time scales.

\begin{tabular}{ccc}
\hline \hline $\begin{array}{c}\text { angular } \\
\text { resolution } \\
{[\mathrm{mas}]}\end{array}$ & $\begin{array}{c}\text { linear scale } \\
\text { scale } \\
{[\mathrm{mpc}]=10^{-3} \mathrm{pc}}\end{array}$ & $\begin{array}{c}\text { orbital } \\
\text { time scale } \\
\text { [years] }\end{array}$ \\
\hline 1000 & 40 & 440 \\
100 & 4 & 14 \\
60 & 2.4 & 6.5 \\
30 & 1.2 & 2.3 \\
15 & 0.6 & 0.8 \\
\hline \hline
\end{tabular}

Table 2. Expected relativistic periastron shift $\Delta \varphi$ for $100 \%$ of the mass contained in a single $\mathrm{BH}$, calculated for different semi-major axis $a$ and eccentricities $e$.

\begin{tabular}{cccc}
\hline \hline$a[\mathrm{mas}]$ & $a[\mathrm{mpc}]$ & $\begin{array}{c}\Delta \varphi \\
e=0.5\end{array}$ & $\begin{array}{c}\Delta \varphi \\
e=0.9\end{array}$ \\
\hline 60 & 2.4 & $-5.0^{\prime}$ & $-20^{\prime}$ \\
30 & 1.2 & $-10^{\prime}$ & $-39^{\prime}$ \\
20 & 0.8 & $-15^{\prime}$ & $-59^{\prime}$ \\
15 & 0.6 & $-20^{\prime}$ & $-1.3^{\circ}$ \\
\hline \hline
\end{tabular}

Center one can compare it to the case of other measured periastron advances. In the case of Mercury, the measured relativistic shift is of the order of $\approx 0.1$ arcsec per revolution. For the Hulse-Taylor Pulsar PSR B1913+16 the shift is $\approx 13$ arcsec per revolution (Taylor 1993). Therefore, the expected relativistic shifts for stars on orbits with semimajor axes as those listed in Table 2 could be, per revolution, 10 to $10^{2}$ times bigger than that of the Hulse Taylor Pulsar, and $10^{3}$ to $10^{4}$ times bigger than the one of Mercury.

As the precise shape of the orbit will depend on the particular central mass distribution, it is most useful to have a general framework to compute the orbits for a particular choice of central mass distribution. If one wants to include the first order general relativistic contribution, in particular the relativistic periastron advance, one can use the so-called post-Newtonian approximation of General Relativity, which is described in Appendix A.

\section{Extended mass distribution}

In order to study the Newtonian orbital shift we consider the simplest case of a spherically symmetric mass distribution. We assume that a given star can enter the extended mass distribution, and neglect any non-gravitational interaction. We also neglected the influence of lensing (see Sect. 4.4).

We assume that the total mass of the central compact distribution amounts to $2.9 \times 10^{6} M_{\odot}$.

As a consequence of the spherical symmetry of the considered mass distribution, the (Newtonian) gravitational force on a given star depends only on the enclosed mass within the radius corresponding to the position of the star.

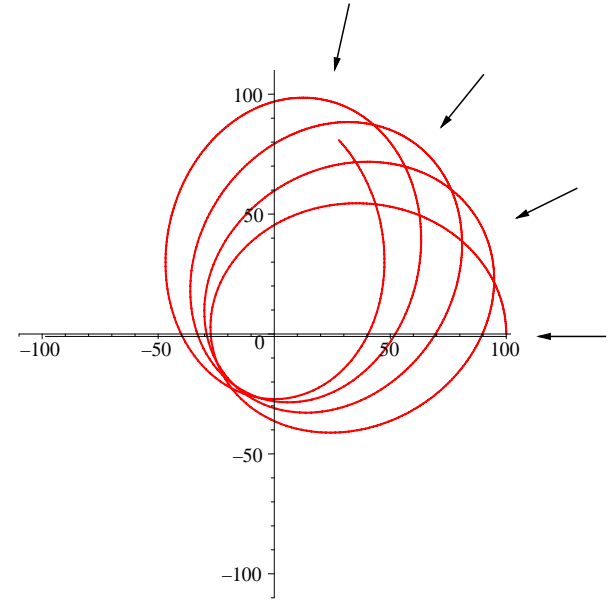

Fig. 2. Example for prograde relativistic periastron advance. Units are given in gravitational length scales $G M / c^{2}$. Apoastron locations are indicated.

Therefore, as it moves towards the center of forces, the gravitational force and hence the curvature of the orbit is smaller as compared with the case in which the whole mass is concentrated within a radius smaller than the periastron radius of the stellar orbit. This leads to orbits with a retrograde orbital shift - that is a shift in the opposite direction as compared with the relativistic orbital shift.

\subsection{Simple model: Uniform density sphere}

Jiang \& Lin (1985) present a simple analytical treatment of the orbits of a test particle which is allowed to enter into the inner region of a sphere with uniform matter distribution. Only the Newtonian gravitational force is considered. In this case, the potential is given by

$\phi(r)= \begin{cases}\frac{G M}{2 R^{3}} r^{2}-\frac{3 G M}{2 R} & r \leq R, \\ -\frac{G M}{r} & r>R,\end{cases}$

where $R$ is the radius of the sphere of total mass $M$. They have shown, that for a given $M$ and $R$ the resulting orbit precession is given by

$\Delta \varphi=2 \arccos \left[\Xi_{1}(e, a)\right]+\arcsin \left[\Xi_{2}(e, a)\right]-\frac{\pi}{2}$,

with

$\Xi_{1}(e, a)=\frac{1}{e}\left[\frac{a}{R}\left(1-e^{2}\right)-1\right]$,

$\Xi_{2}(e, a)=\frac{\frac{2}{R^{2}}-B}{\sqrt{B^{2}+4 A}}$

and

$A:=-\frac{1}{a R^{3}\left(1-e^{2}\right)}, \quad B:=\frac{1}{a^{2}\left(1-e^{2}\right)}\left(\frac{3 a}{R}-1\right)$.

Here we have rewritten the results of Jiang \& Lin (1985) in terms of the semi-major axis $a$ and the eccentricity $e$ of the outer Keplerian orbit. 
Table 3. Expected Newtonian periastron shift $\Delta \varphi$ for $100 \%$ of the mass distributed in a circular sphere of radius $R$ and constant density for two different orbits: (1) semi-major axis $a=60$ mas $=2.4 \mathrm{mpc}, e=0.5$ and (2) semi-major axis $a=15$ mas $=0.6 \mathrm{mpc}, e=0.5$.

\begin{tabular}{cccccc}
\hline \hline & Orbit(1) & & \multicolumn{3}{c}{ Orbit(2) } \\
$R$ & $\begin{array}{c}R \\
{[\mathrm{mpc}]}\end{array}$ & $\begin{array}{c}\Delta \varphi \\
{[\mathrm{deg}]}\end{array}$ & $\begin{array}{c}R \\
{[\mathrm{mas}]}\end{array}$ & $\begin{array}{c}R \\
{[\mathrm{mpc}]}\end{array}$ & $\begin{array}{c}\Delta \varphi \\
{[\mathrm{deg}]}\end{array}$ \\
\hline$<30$ & $<1.2$ & 0 & $<7.5$ & $<0.3$ & 0 \\
35 & 1.4 & 30 & 10 & 0.4 & 56 \\
40 & 1.6 & 56 & 12.5 & 0.5 & 92 \\
50 & 2.0 & 93 & 15 & 0.6 & 120 \\
\hline \hline
\end{tabular}

Some expected periastron shifts calculated as a pure Newtonian effect are listed in Table 3. The shifts are substantial: several $10^{\circ}$ - even after a single revolution. In the (close to) non-relativistic Newtonian limit we tested our post-Newtonian numerical calculations against the results of this analytical formula.

\subsection{Plummer-like model plus black hole: Post-Newtonian Treatment}

We now model the central mass distribution as composed by a central BH ("point mass") plus a spherically symmetric matter distribution, phenomenologically parameterized by means of a Plummer-like distribution of the form

$\rho_{\alpha}(r)=\frac{1}{\left[1+\left(r / r_{\mathrm{c}}\right)^{2}\right]^{\alpha / 2}}$,

where $r_{\mathrm{c}}$ and $\alpha$ are constants so that at $r=r_{\mathrm{c}}$ the density has decreased by a factor $2^{-\alpha / 2}$ from its central value (see also Fig. 3).

In the following we use $\lambda_{\mathrm{p}}$ as the fraction of the total mass which is contained in the compact unresolved component and $\lambda_{\mathrm{e}}=1-\lambda_{\mathrm{p}}$ the fraction of mass that is extended. The normalization in the following Eqs. (10) and (12) is chosen such that the enclosed mass $M(r) \rightarrow M$ for $r \rightarrow \infty$ and $M(r) \rightarrow \lambda_{\mathrm{p}} M$ for $r \rightarrow 0$.

As required the condition that $\rho \rightarrow 0$ for $r \rightarrow \infty$ is also fulfilled. The mass density is then given by

$\rho(r):=M\left[\lambda_{\mathrm{p}} \delta^{(3)}(\boldsymbol{r})+\lambda_{\mathrm{e}} \frac{\rho_{\alpha}(r)}{4 \pi \int_{0}^{\infty} \zeta^{2} \rho_{\alpha}(\zeta) \mathrm{d} \zeta}\right]$

where $M$ is the total mass of the system. The mass inside a sphere of radius $r$ is given by

$$
\begin{aligned}
M(r) & =4 \pi \int_{0}^{r} \zeta^{2} \rho(\zeta) \mathrm{d} \zeta \\
& =M\left[\lambda_{\mathrm{p}}+\lambda_{\mathrm{e}} \frac{\int_{0}^{r} \zeta^{2} \rho_{\alpha}(\zeta) \mathrm{d} \zeta}{\int_{0}^{\infty} \zeta^{2} \rho_{\alpha}(\zeta) \mathrm{d} \zeta}\right]
\end{aligned}
$$

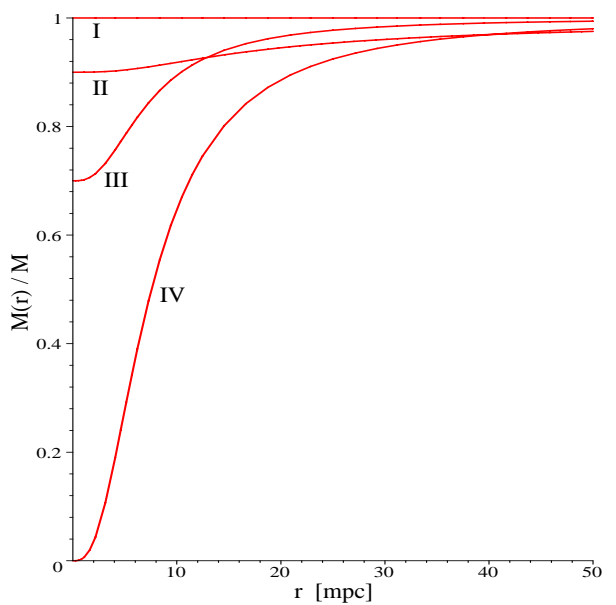

Fig. 3. Example of possible enclosed mass distributions. Here $100 \%, 90 \%, 70 \%$, and $0 \%$ of the measured total mass is contained in a BH, for curves I, II, III, and IV, respectively. For the cases III, and IV the remaining fraction is in a cluster of dark mass following a Plummer distribution with $\alpha=5$ and $r_{\mathrm{c}}=5.8 \mathrm{mpc}$. For case II $\alpha=3$ and $r_{\mathrm{c}}=10 \mathrm{mpc}$.

The potential can be computed from the enclosed mass curve using

$\phi(r)=-G \int_{r}^{\infty} \frac{M(\zeta)}{\zeta^{2}} \mathrm{~d} \zeta$.

In Fig. 3 we show examples of possible enclosed mass curves for a core radius $r_{\mathrm{c}}=5.8 \mathrm{mpc}, \alpha=5$, and $\lambda_{\mathrm{p}}=0,0.7$ and 1 .

With this information, we numerically solve the postNewtonian equations of motion (A.7). As an example we have computed the orbits in Fig. 4 for several values of $\lambda_{\mathrm{p}}$ and the following initial conditions:

$\boldsymbol{x}_{0}=(10,0)[\mathrm{mpc}], \quad \boldsymbol{v}_{0}=(0,500)\left[\mathrm{km} \mathrm{s}^{-1}\right]$,

which correspond to an eccentricity of $e \approx 0.80$ and are similar to those of the star $S 2$ (as seen projected onto the plane of the sky) close to the position of Sgr A* (Eckart et al. 1997). For simplicity we have assumed that the orbits are contained in the plane of the sky.

To find the orbits and the corresponding shifts, we first compute the mass and potential profiles as given above. Then, we use a fourth-fifth order Runge-Kutta method to solve (A.7) with the initial conditions (14) for the orbit $\boldsymbol{x}(t)$. Then we look for two successive apoastron positions, i.e. points along the orbit having a maximum value of $|\boldsymbol{x}|$. From these two points, the corresponding angular shift is finally computed. In principle, one could directly compute the shifts using the newtonian angular integral for an spherically symmetric system $\Delta \varphi=\int_{r_{\mathrm{p}}}^{r_{\mathrm{a}}} \mathrm{d} r \frac{l}{r^{2}}\left[2 E-\frac{l^{2}}{r^{2}}-2 \phi\right]^{-1 / 2}$ and its postNewtonian generalization. This was, however, dificult to implement in practice due to the singularities of the integrand at the end points.

As expected, the motion of the star is already dominated by very small extended mass distributions, which is 

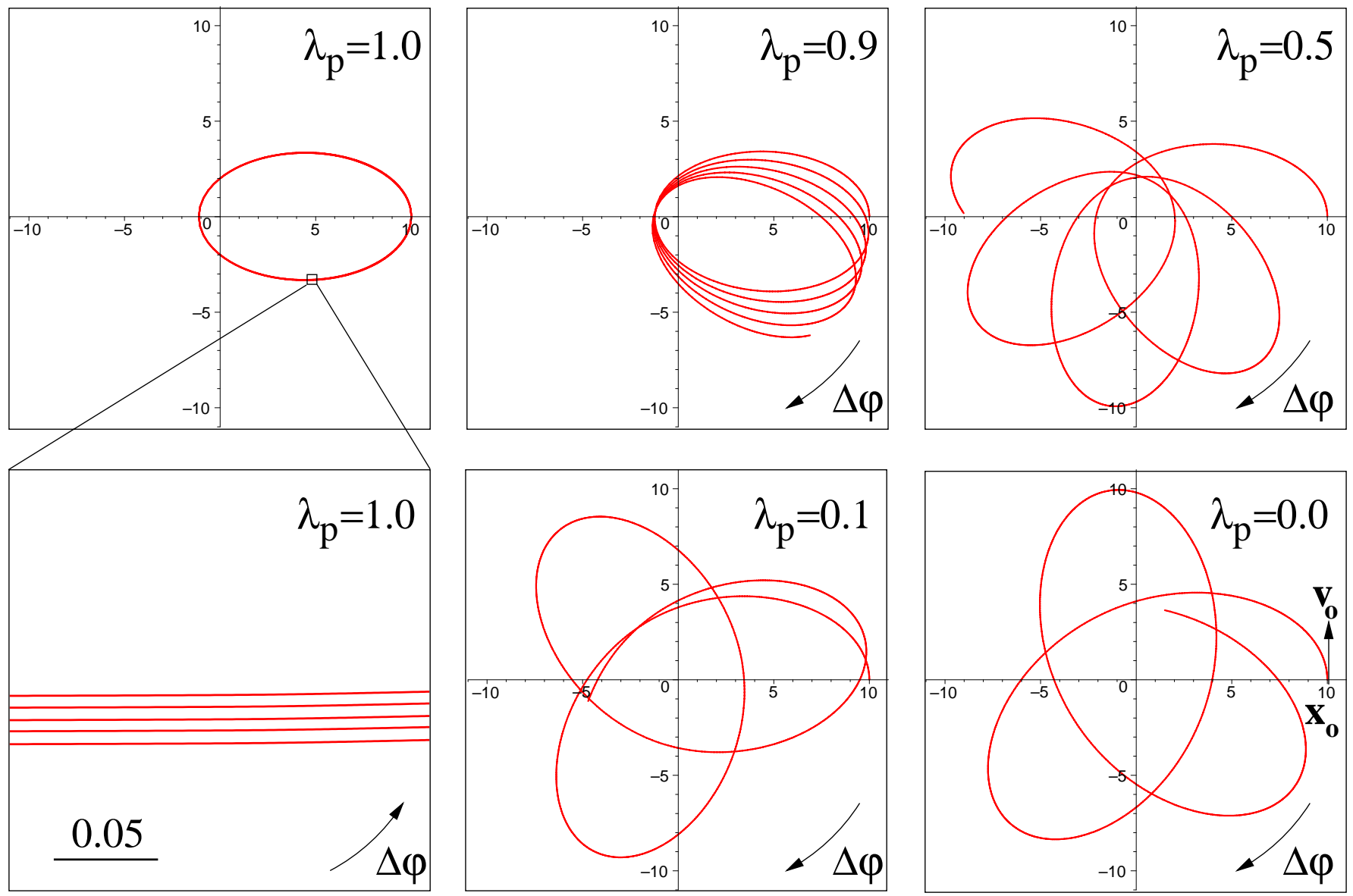

Fig. 4. Post-Newtonian orbits for different values of compact mass parameter $\lambda_{\mathrm{p}}$ for a star with initial conditions given in Eq. (14). The axes and scale bar are labeled in units of mpc.

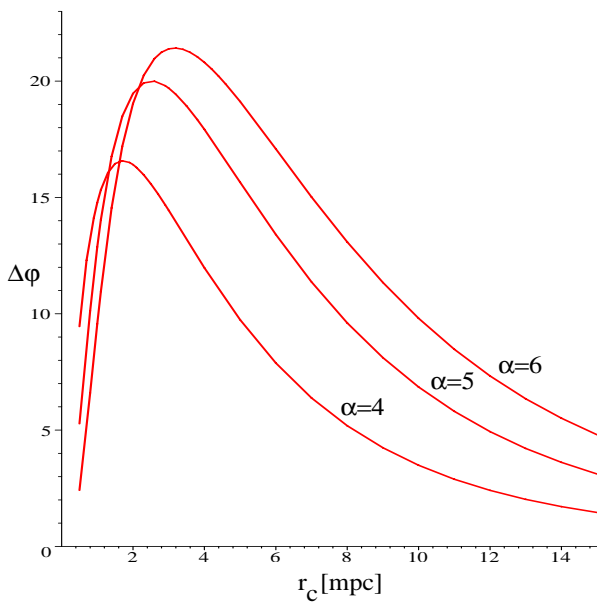

Fig. 5. Orbital shifts in degrees as a function of $r_{\mathrm{c}}$, for $\alpha=$ $4,5,6$, and $\lambda_{\mathrm{p}}=0.8$ for a star with initial conditions given in Eq. (14).

essentially a pure Newtonian effect. In this example the differences between the Newtonian and post-Newtonian orbital shifts are less than $1 \%$.

In Fig. 5 we show the orbital shifts as a function of $r_{\mathrm{c}}$, for $\alpha=4,5,6$, and $\lambda_{\mathrm{p}}=0.8$ for a star with initial conditions given in Eq. (14). This plot demonstrates that there is a maximum orbital shift depending on the properties of the mass distribution. Decreasing $r_{\mathrm{c}}$ from infinity to smaller values, the amount of mass that is resolved by the stellar orbit between the apoastron and periastron increases which results in an increasing orbital shift. Then the amount of resolvable mass decreases again as the extent of the mass distribution shrinks below the periastron radius. The maximum orbital shift is reached faster by the sharper edged mass distributions (i.e. for larger values of $\alpha$ ). This behavior represents a degeneracy in the sense that independent of $\alpha$ there are in general two different radii $r_{\mathrm{c}}$ at which a given observable periastron shift occurs. Therefore, (for a given fraction of unresolved mass $\left.\lambda_{\mathrm{p}}\right)$ a minimum of two stars on different orbits is required to derive the compactness (shape of the distribution described by $\alpha$ ) of the central mass concentration. Three stars on different orbits (i.e. with different orbital energy $E$ or angular momentum $l$ ) are necessary in order to unambiguously solve for all three parameters $\lambda_{\mathrm{p}}, \alpha$, and $r_{\mathrm{c}}$.

We also show in Fig. 6 for the same initial conditions as given in Eq. (14) the case in which just a fraction of less than $1 \%$ of the total mass is extended. For $\lambda_{p}=1$, i.e. just a central $\mathrm{BH}$ the orbital shift reduces to that corresponding to Eq. (1) which amounts to $0.074^{\circ}=4.4^{\prime}$ per revolution. These prograde shifts are shown in Fig. 6 as negative 


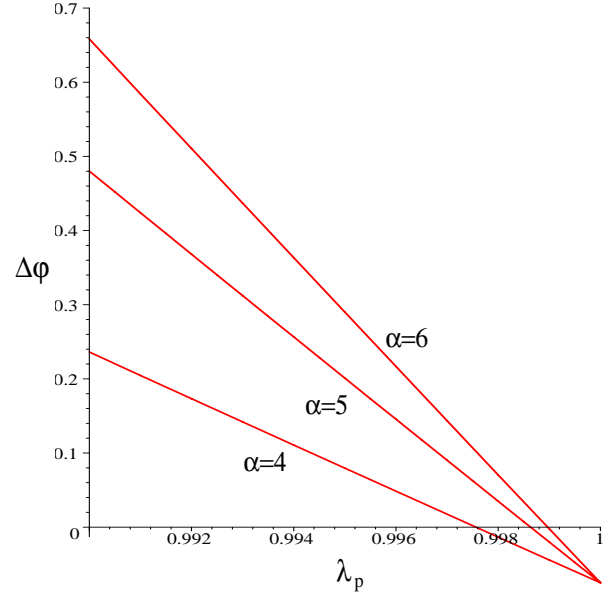

Fig. 6. Post-Newtonian periastron shifts in degrees for different values of compact unresolved mass parameter $\lambda_{\mathrm{p}}$. Here $r_{\mathrm{c}}=5.8 \mathrm{mpc}$.

Table 4. Fraction of extended mass required to compensate the relativistic shift, for different initial conditions and values of $r_{\mathrm{c}}$. Here $\alpha=5$.

\begin{tabular}{cccc}
\hline \hline $\begin{array}{c}r_{0} \\
{[\mathrm{mpc}]}\end{array}$ & $\begin{array}{c}v_{0} \\
{\left[\mathrm{~km} \mathrm{~s}^{-1}\right]}\end{array}$ & $\begin{array}{c}r_{\mathrm{c}} \\
{[\mathrm{mpc}]}\end{array}$ & $\begin{array}{c}\text { fraction of } \\
\text { ext. mass }\end{array}$ \\
\hline 10 & 500 & 5.8 & $0.14 \%$ \\
1 & 500 & 5.8 & $75 \%$ \\
1 & 500 & 2.0 & $50 \%$ \\
1 & 1500 & 5.8 & $42 \%$ \\
1 & 1500 & 2.0 & $11 \%$ \\
\hline \hline
\end{tabular}

values. The transition from a prograde (essentially relativistic) orbital shift and the retrograde shift due to the extended mass distribution occurs at $\lambda_{\mathrm{p}} \approx 0.9976,0.9986$ and 0.9990 for $\alpha=4,5$ and 6 , respectively. That corresponds to fractions of only $0.24 \%, 0.14 \%$ and $0.10 \%$ of the total mass.

Therefore, for S2-like orbits, only a fraction of the order of $0.1 \%$ of the total mass is needed to be extended in order to compensate the relativistic orbital shift. This amounts to about $3000 M_{\odot}$. Unless there is a stellar cusp (see Alexander 1999 and discussion in Sect. 4.1) this is about 1000 times larger than the expected density from the $r_{\mathrm{c}} \sim 0.38 \mathrm{pc}$ stellar cluster in the same volume contained in a sphere of radius $r_{\mathrm{c}} \sim 5.8 \mathrm{mpc}$. As demonstrated in Table 4 the fraction of mass required to compensate the relativistic shift is larger for smaller or more eccentric orbits.

\section{The link to observations}

The current measurements have convincingly proven that a mass of $(2.9 \pm 0.4) \times 10^{6} M_{\odot}$ (Eckart \& Genzel 1996, 1997; Genzel et al. 1997, 2000; Ghez et al. 1998, 2000) is enclosed within a central sphere of radius $<10 \mathrm{mpc}$ $\left(0.25^{\prime \prime}\right)$. Most recent observational capabilities and especially those that will become available in the very near

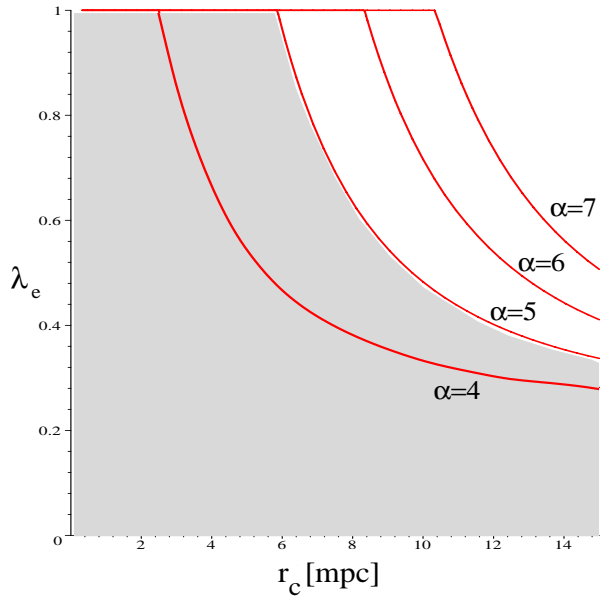

Fig. 7. Limits for the parameter $\lambda_{\mathrm{e}}=1-\lambda_{\mathrm{p}}$ describing the fraction of extended mass for different values of $\alpha$. For $\alpha=5$ the range of allowed values for $\lambda_{\mathrm{e}}$ and $r_{\mathrm{c}}$ is grey-shaded.

future will allow to determine observationally the compactness of that mass and the properties of the distribution of any extended fraction of it.

\subsection{How compact is the central mass?}

Considering the measurement uncertainties and the uncertainties in the $r \sim 0.38 \mathrm{pc}$ core radius of the visible cluster, the current data do not exclude that a fraction of the central compact mass is extended. Such an additional component could be an extended dark mass consisting of neutron stars, stellar black holes or white dwarfs (e.g. Morris 1993; Saha et al. 1996; Haller et al. 1996). However, a very steep outer density distributions of such a dark cluster (e.g. a Plummer-like model with $\alpha \geq 5$; see Eq. (9)) is inconsistent with any known observed dynamical system as well as the results of current physical models, including those of core-collapsed clusters (see the discussion by Genzel et al. 1997). Maoz 1998 points out that the only possible - although highly implausible - alternatives to a central $\mathrm{BH}$ are a concentration of heavy bosons and a compact cluster of light $\left(<0.005 M_{\odot}\right)$ black holes. The current measurement uncertainties, however, would also allow a more shallow distribution containing a small fraction of the mass enclosed within the central $10 \mathrm{mpc}$ (e.g. $\lambda_{\mathrm{e}} \leq 0.2,5 \mathrm{mpc}<r_{\mathrm{c}}<100 \mathrm{mpc}, \alpha<5$ ). Such a more shallow mass distribution would be provided by a possible stellar cusp. Alexander (1999) finds that in a compilation of high angular resolution NIR imaging data sets the likelihood curves for sizes of a possible cusp peak at $r_{\mathrm{c}}<2.5^{\prime \prime}(100 \mathrm{mpc})$.

In order to investigate the possible parameter region of our mass distribution model, we analyze the extreme case by setting $M=3.3 \times 10^{6} M_{\odot}$. Next, we impose that the mass at $r=13 \mathrm{mpc}$ has to be greater than $2.5 \times 10^{6} M_{\odot}$. 
For these conditions we solve Eq. (12) for the maximum $\lambda_{\mathrm{e}}$, which we call $\lambda_{\mathrm{e}}^{\mathrm{m}}$, allowed for each $\alpha$ and $r_{\mathrm{c}}$. We obtain

$$
\lambda_{\mathrm{e}}^{\mathrm{m}}=\frac{1-\frac{2.5}{3.3}}{1-\frac{\int_{0}^{13} \zeta^{2} \rho_{\alpha}(\zeta) \mathrm{d} \zeta}{\int_{0}^{\infty} \zeta^{2} \rho_{\alpha}(\zeta) \mathrm{d} \zeta}} .
$$

Since the condition $\lambda_{\mathrm{e}}<1$ has to be fulfilled as well, the real maximum is given by

$\lambda_{\mathrm{e}}^{\max }=\min \left(1, \lambda_{\mathrm{e}}^{\mathrm{m}}\right)$.

As a result, for each $\alpha$, the allowed parameter region lies below the corresponding curve in Fig. 7. Thus, for instance, for $\lambda_{\mathrm{e}}=1$ (just extended mass, no $\mathrm{BH}$ ) and for $\alpha=5$, the maximum allowed value for $r_{\mathrm{c}}$ is $\sim 5.86 \mathrm{mpc}$, in agreement with the results of Genzel et al. (1998).

\subsection{Can the periastron shifts be measured?}

In the near future, observations using large diameter telescopes in combination with adaptive optics and especially interferometric methods [using Keck I and Keck II ${ }^{1}$ (10 m; Booth et al. 1999, Swanson et al. 1997), the ESO very Large Telescope (VLT, $8.2 \mathrm{~m}$ ) and $\mathrm{VLTI}^{2}$ (von der Lühe et al. 1997; Eckart et al. 1997; Glindemann \& Lévêque 2000), as well as the Large Binocular Telescope ${ }^{3}$ (LBT, $2 \times 8.4 \mathrm{~m}$ on a $14.4 \mathrm{~m}$ baseline; Hill et al. 1998; Angel et al. 1998)] will have an even higher angular resolution and larger point source sensitivity than currently available. The Keck and VLT interferometers will achieve an angular resolution of a few milliarcseconds in the nearinfrared over a small field of view of the order of $1^{\prime \prime}$. As a Fizeau-type interferometer the LBT interferometer, however, will combine high angular resolution (20 to 30 mas in the NIR) with an exceptionally large field of view (FOV) of the order of $30^{\prime \prime}$ to $60^{\prime \prime}$.

In order to give an impression of the accuracy with which near future measurements will allow us to investigate stellar orbits near $\operatorname{Sgr} \mathrm{A}^{*}$ we show in Fig. 8 a simulated LBT snapshot image of the central $1.2^{\prime \prime} / \times 1.2^{\prime \prime}$. The image shows stars brighter than $K=15$ as seen with the LBT interferometer at the epoch 2000. A source corresponding to the possible identification of a NIR counterpart of Sgr A* has been included as well (Genzel et al. 1997). Assuming (conservatively) that the relative position of stars can be determined to about $1 / 30$ of the achieved angular resolution, the accuracy will be of the order of 1 mas. Given the large FOV the positions and proper motions of stars in the entire central stellar cluster can be measured. The accuracy can even be improved if a large number $N$ of reference stars is used. The late type stars are especially well suited as reference stars, since they have on average much lower proper motions $\left(<100 \mathrm{~km} \mathrm{~s}^{-1}\right.$ for $\left.r>10^{\prime \prime}\right)$ as compared to the bright He I

\footnotetext{
1 http://huey.jpl.nasa.gov/keck/

2 http://www.eso.org/projects/vlti/

3 http://medusa.as.arizona.edu/lbtwww/
}

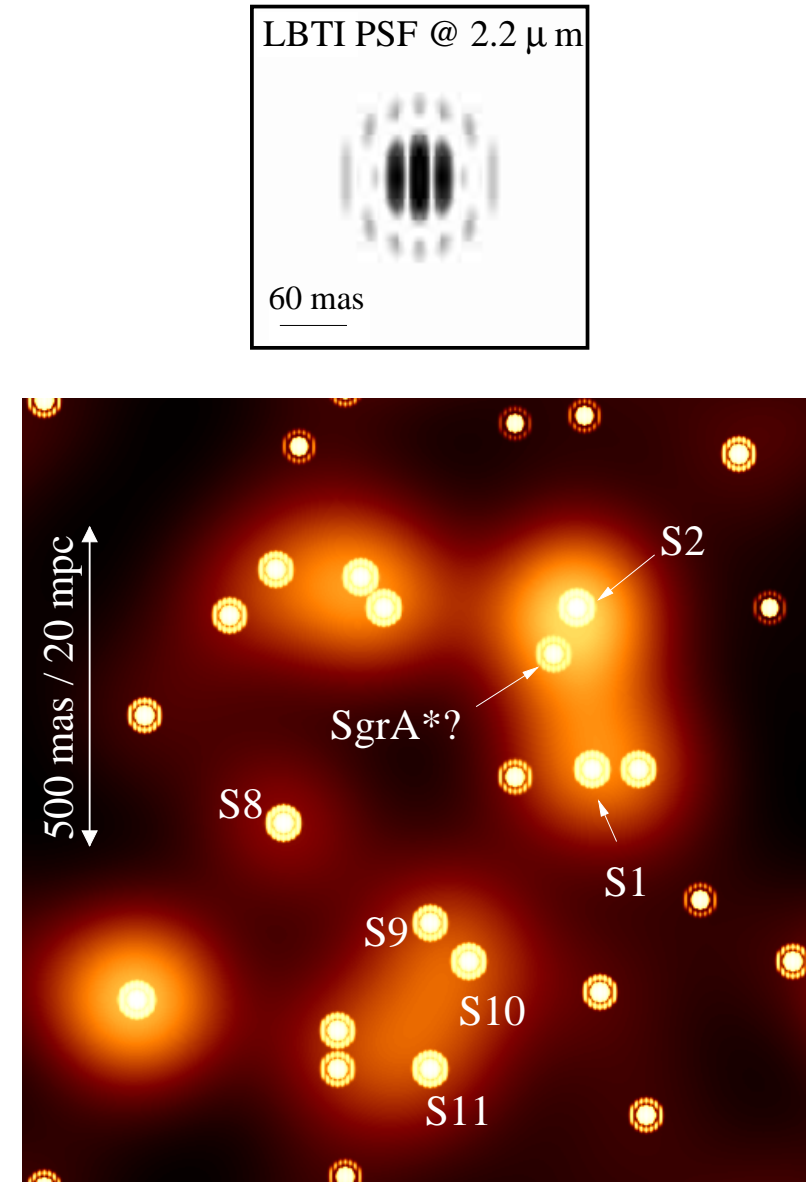

Fig. 8. Central arcsecond as seen in a snapshot with the LBT interferometer at the epoch 2000. Corresponding to the visibility above the horizon the final synthesized beam will have a smaller ellipticity and side lobe level than the snapshot PSF. For the simulation we took into account stars with $K<15$. We assumed a single aperture Strehl ratio of $50 \%$ which gives rise to the halos around the stars.

stars in the central few arcseconds $\left(\sim 200-300 \mathrm{~km} \mathrm{~s}^{-1}\right.$ for $\left.r<8^{\prime \prime}\right)$. Therefore, the wide FOV of the LBT interferometer can be used to reference the positions especially of the central stars to several hundred late type stars. The positional accuracy will be increased by approximately $\sqrt{N}$ and could finally be of the order of $100 \mu$ as (or even better; Jaroszyǹski 1999 quotes $\sim 20 \mu$ as for the Keck interferometer; see also Quirrenbach 2000). This implies that the relativistic periastron shifts listed in Table 2 could all be measured with an accuracy of $1 \sigma$ to $3 \sigma$ per revolution. Combined with the orbital time scales of only about 1 year for orbits with semi-major axes of the order of $1 \mathrm{mpc}$ (see Table 1) this means that if only the relativistic periastron shift due to a single compact mass would be important a significant prograde shift could be determined after only a few years or revolutions. Since a retrograde shift due to a possible extended mass can be of the order of $10^{\circ}$ or more, it could be measured after only a single revolution since the star will reach its apoastron at a significantly different position than before. 

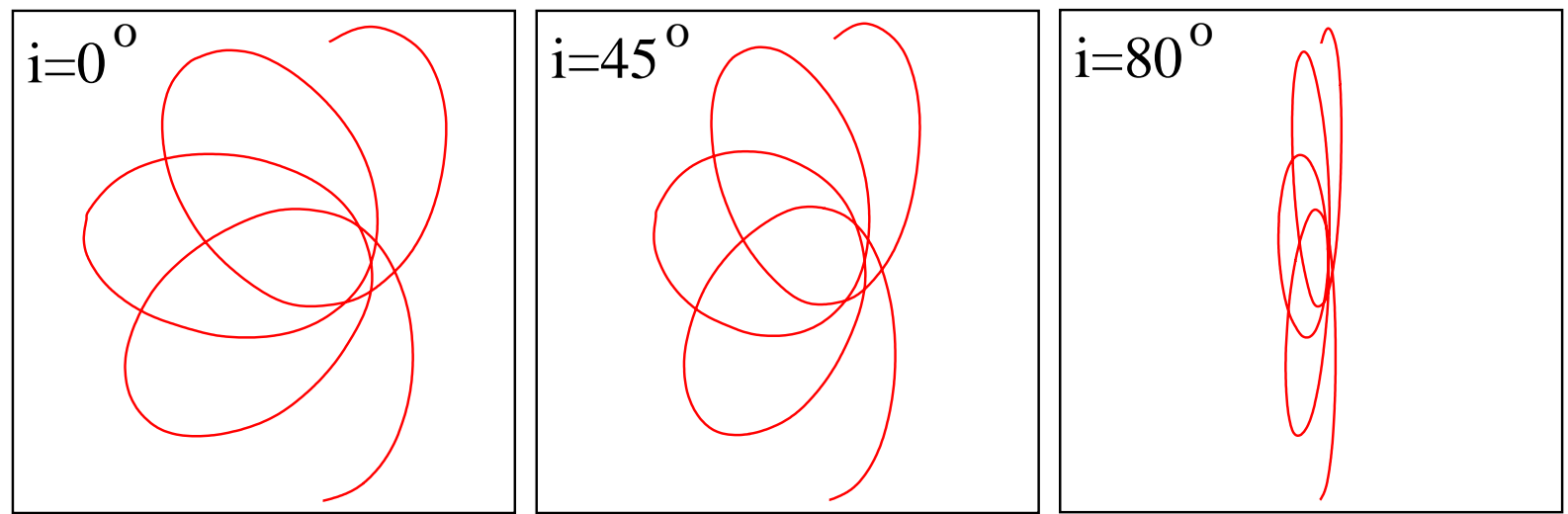

Fig. 9. Orbital shift for an S2-like star at three different inclinations. The initial conditions are the same as in Fig. 4.

\subsection{Inclination of the stellar orbits}

In case a periastron shift can be obtained this offers an independent method of measuring the inclination of the stellar orbit. Since the shift occurs in the orbital plane the positions at which the star reaches its periastron are located on a circle (see Fig. 9). With a minimum of three periastron locations or a high signal to noise measurement of the orbit between two periastron locations the inclination of the orbital plane can be determined. This will allow us to derive the inclination corrected value for any observed periastron shift. This determination of the inclination will also be of great value in those cases where it is difficult to measure the radial (Doppler) component of the stellar motion. Knowledge of the sky positions, proper motions and the orbital inclination then allow a determination of the stellar orbit (with exception of the rotational sense).

\subsection{The effect of lensing}

Lensing may lead to flux amplifications or the occurrence of multiple images over a limited period of time. Combined with the effects of inclination (e.g. Fig. 9) especially the possible multiplicity of images could make it difficult to interpret the observations. Therefore, the influence of lensing on trajectory measurements of the central high velocity stars has to be discussed. Wardle \& Yusef-Zadeh (1992), Alexander \& Sternberg (1999), and Alexander \& Loeb (2000) discussed the influence of the Galactic Center $\mathrm{BH}$ alone on the stars in the inner stellar cluster. The angular size of the Einstein radius, $\theta_{\mathrm{E}}$, is given by

$\theta_{\mathrm{E}} \sim 17$ mas $\times D_{8}^{-1}\left(M_{3.0} d_{\mathrm{pc}}\right)^{1 / 2}$

(see Alexander \& Sternberg 1999). Here $M_{3.0}$ is the BH mass in units of $3.0 \times 10^{6} M_{\odot}, D_{8}$ is the distance to the Galactic Center in units of $8 \mathrm{kpc}$, and $d_{\mathrm{pc}}$ is the lenssource distance in parsec. This linear approximation for small light-bending angles holds as long as the Einstein radius $R_{\mathrm{E}}$ is much larger than the Schwarzschild radius of the $\mathrm{BH}$. The observations are carried out with a given limiting angular resolution $\psi$. If $\theta_{\mathrm{E}}<\psi$, the two images are unresolved and the lensed source simply appears as a micro-lensing event. For a given angular resolution $\psi$,
Alexander \& Sternberg (1999) derive a maximal lenssource distance $d_{\mu}$ for micro-lensing

$d_{\mu}=\frac{D_{8}^{2}}{M_{3.0}}\left(\frac{\psi}{17 \mathrm{mas}}\right)^{2} \mathrm{pc}$

such that $\theta_{\mathrm{E}}>\psi$ for $d>d_{\mu}$ and the multiple stellar images can be resolved. For angular resolutions achievable with the LBT in the NIR the lensing events for stars within the central parsec will therefore not be resolved. This will be true for most of the visible central stellar cluster and especially in the entire Sgr A* central cluster of high velocity stars. Even for angular resolutions as high as 2.5 mas as achievable in the near future with the VLTI one finds $d_{\mu}=0.021 \mathrm{pc}$ which corresponds to $0.5^{\prime \prime}$ on the angular scale. This shows that although lensing of central stars may in fact occur it will most likely have no major perturbing influence on the determination of their orbital motions. The situation may improve even further if the amount of mass contained in the central BH is decreased. For $\lambda_{\mathrm{p}}=0.5$ the entire central stellar cluster over a diameter of about $2^{\prime \prime}$ at an angular resolution of $\sim 2.5$ mas is not affected by the occurrence of resolved multiple images of its cluster members. Since the high magnification events near the Einstein radius will have a duration of only up to a few months one should be able to clearly discriminate the corresponding background sources from central stars that are truly orbiting the Galactic Center BH.

\section{Summary and conclusion}

The Galactic Center is the closest and, besides NGC 4258 (Greenhill et al. 1995; Myoshi et al. 1995), the most convincing candidate for a massive nuclear BH (Maoz 1998). We have investigated what the properties of stellar orbits close to this central mass are and how this is linked to current and (near) future observational capabilities. Our results can be summarized in the following points:

- The orbital shift can be due to relativistic effects, resulting in a prograde shift, and due to a possible extended mass distribution, producing a retrograde shift. With the increased measurement accuracy expected 
for NIR imaging in the near future, both kinds of shifts will be measurable, specially for stars (yet to be found) with orbits within less than $1 \mathrm{mpc}$ from the center. Those stars will have orbital periods of the order of one year and less;

- In case a orbital periastron shift is observed the inclination of the stellar orbit and hence the inclination corrected value of the shift can be determined;

- The retrograde Newtonian shift may partially or completely compensate the relativistic shift. We have found that in the case for S2-like stars, the retrograde shift, induced by a possible extended mass distribution, is likely to be dominant and small fractions of extended mass of typically $0.1 \%$ of the total mass (of about $3 \times 10^{6} M_{\odot}$ within $<10 \mathrm{mpc}$ ) are sufficient to compensate the prograde relativistic contribution. The fraction of mass required to compensate the relativistic shift is larger for smaller or more eccentric orbits. Therefore, even if a prograde relativistic shift is measured it may still be influenced by a weak underlying extended mass contribution, resulting in a smaller observed prograde shift than predicted by General Relativity for a single BH (cf. Eq. (1) and Table 2);

- Our calculations show that the expected orbital shifts are degenerated with respect to the three parameters $\lambda_{\mathrm{p}}, \alpha$, and $r_{\mathrm{c}}$ which we used to describe the extended mass contribution. Limits of $\lambda_{\mathrm{p}}$ and $r_{\mathrm{c}}$ as a function of $\alpha$ were presented in Sect. 4.1. For a given $\alpha$ and $\lambda_{\mathrm{p}}$ there are in general two different radii $r_{\mathrm{c}}$ at which a given observable periastron shift occurs. All three parameters, however, can be found by analyzing the inclination corrected orbital parameters of 3 stars with different orbital energy $E$ or angular momentum $l$;

- Lensing of stars in the visible cluster by the central $\mathrm{BH}$ will not perturb measurements of the stellar orbits. Distant background stars that may happen to be lensed by central stellar lensed in the shear field of the $\mathrm{BH}$ can be discriminated against orbiting sources at the center since the duration of these lensing events is only a few months.

\section{Appendix A: The post-Newtonian approximation}

The post-Newtonian approximation (see Weinberg 1972; Will 1993; Schneider 1996) applies to systems of slowly moving particles (with respect to the velocity of light) bound by gravitational forces. In such systems the typical values of the Newtonian potential $\bar{\phi} \sim \frac{G \bar{M}}{\bar{r}}(\bar{M}$ and $\bar{r}$ are the typical mass and distance between particles) is of the same order of magnitude as the typical squared velocities of the particles $\bar{v}^{2}$ (e.g. for a test particle in a circular orbit of radius $r$ around a central mass $M$ we actually have $\left.v^{2}=G M / r\right)$. The post-Newtonian approximation is based on an expansion of the quantities involved in the determination of the trajectories of the particles, in powers of the small parameter $v$, taking into account that $O(\phi) \sim O\left(v^{2}\right)$, and keeping only the first post-Newtonian correction in a consistent way. In this section we follow the conventions and notation of Weinberg (1972) and use $c=1$.

Within this approximation, and modeling the matter distribution as static and pressureless, the metric tensor is given by

$$
\begin{aligned}
g_{00} & =-1-2 \phi-2 \phi^{2}+O\left(v^{6}\right), \\
g_{0 i} & =0+O\left(v^{5}\right), \\
g_{i j} & =\delta_{i j}(1-2 \phi)+O\left(v^{4}\right),
\end{aligned}
$$

where

$\phi:=-G \int \mathrm{d}^{3} x^{\prime} \frac{\rho\left(r^{\prime}\right)}{\left|\boldsymbol{x}-\boldsymbol{x}^{\prime}\right|}$.

The geodesic equation, i.e. the equation of motion for free test particles, then reads

$\frac{\mathrm{d} \boldsymbol{v}}{\mathrm{d} t}=-\boldsymbol{\nabla}\left(\phi+2 \phi^{2}\right)+4 \boldsymbol{v}(\boldsymbol{v} \cdot \boldsymbol{\nabla}) \phi-v^{2} \boldsymbol{\nabla} \phi+O\left(\frac{v^{6}}{r}\right)$.

The total gravitating mass of the system is given by $M=$ $\int \mathrm{d}^{3} x \rho$.

For a spherically symmetric matter distribution one has

$\boldsymbol{\nabla} \phi=\frac{G M(r)}{r^{3}} \boldsymbol{r}, \quad M(r)=4 \pi \int_{0}^{r} r^{2} \rho(r) \mathrm{d} r$,

so that (A.5) becomes

$\frac{\mathrm{d} \boldsymbol{v}}{\mathrm{d} t}=-\frac{G M(r)}{r^{3}}\left[\left(1+4 \phi+v^{2}\right) \boldsymbol{r}-4 \boldsymbol{v}(\boldsymbol{v} \cdot \boldsymbol{r})\right]$.

In particular, for a compact mass distribution of total mass $M$ we find $\phi=-\frac{G M}{r}$ and

$\frac{\mathrm{d} \boldsymbol{v}}{\mathrm{d} t}=-\frac{G M}{r^{3}}\left[\left(1-4 \frac{G M}{r}+v^{2}\right) \boldsymbol{r}-4 \boldsymbol{v}(\boldsymbol{v} \cdot \boldsymbol{r})\right]$.

One can verify the correctness of (A.8) by expanding the geodesic equation of a test particle in a Schwarzschild spacetime to the first post-Newtonian order. Compare also with Eq. (37.297) in Schneider (1996) in the limit $\frac{M_{1} / M_{2}}{\left(1+M_{1} / M_{2}\right)^{2}}=\nu \rightarrow 0$, with Eqs. (6.31)-(6.34) in Will (1993) for the case of General Relativity, and with Eq. (39.64) in Misner et al. (1973).

By applying the post-Newtonian equations of motion (A.8) one can derive the relativistic periastron advance for a single $\mathrm{BH}$ as given by Eq. (1) and for more complex spherically symmetric mass distributions as discussed in Sect. 3.

Acknowledgements. GFR is grateful to Friedrich W. Hehl and P.C. Fragile for useful comments and support, to Dirk Puetzfeld for his help, and also to the German Academic Exchange Service (DAAD) for a graduate fellowship (Kennziffer A/98/00829). 


\section{References}

Alexander, T. 1999, ApJ, 527, 835

Alexander, T., \& Sternberg, A. 1999, ApJ, 520, 137

Alexander, T., \& Loeb, A. 2000 [astro-ph/0009404]

Angel, J. R. P., Hill, J. M., Strittmatter, P. A., et al. 1998, Proc. SPIE, 3350, 881

Booth, A. J., et al. 1999, Proc. from ASP Conf. 194., ed. S. Unwin, \& R. Stachnik, 256

Eckart, A., \& Genzel, R. 1996, Nature, 383, 415

Eckart, A., \& Genzel, R. 1997, MNRAS, 284, 576

Eckart, A., Genzel, R., Hofmann, R., et al. 1997, Science with the VLT Interferometer, Proc. of the ESO workshop, held at Garching, Germany, 18-21 June 1996, 259

Fragile, P. C., \& Mathews, J. 2000, ApJ, 542, 328

Genzel, R., Pichon, C., Eckart, A., Gerhard, O. E., \& Ott, T. 2000, MNRAS, 317, 348

Genzel, R., Thatte, N., Krabbe, A., Kroker, H., \& TacconiGarman, L. E. 1996, ApJ, 472, 153

Genzel, R., Eckart, A., Ott, T., \& Eisenhauer, F. 1997, MNRAS, 291, 219

Ghez, A., Klein, B., Morris, M., \& Becklin, E. 1998, ApJ, 509, 678

Ghez, A., Morris, M., Becklin, E. E., Kremenek, T., \& Tanner, A. 2000, Nature, 407, 349

Greenhill, L. J., Jiang, D. R., Moran, J. M., et al. 1995, ApJ, 440,619

Greenhill, L. J., Jiang, D. R., Moran, J. M., et al. 1995, ApJ, 440,619

Haller, J. W., Rieke, M. J., Rieke, G. H., et al. 1996, ApJ, 456, 194

Hehl, F. W., Kiefer, C., \& Metzler, R. 1998, Black Holes: theory and observation, Lect. Notes Phys., 514 (Springer)

Hill, J. M., \& Salinari, P. 1998, Proc. SPIE, 3352, 23

Jaroszyński, M. 1998, Acta Astron., 48, 653

Jaroszyński, M. 1999, ApJ, 521, 591

Jiang, H. X., \& Lin, J. Y. 1985, Am. J. Phys., 53, 694

Kormendy, J., \& Richstone, D. 1995, ARA\&A, 33, 581

Krabbe, A., Genzel, R., Drapatz, S., \& Rotaciuc, V. 1991, ApJ, 382, L19
Krabbe, A., et al. 1995, ApJ, 447, L95

Leonard, P. J. T., \& Merritt, D. 1989, ApJ, 339, 195

Magorrian, J., et al. 1998, AJ, 115, 2285

Maoz, E. 1998, ApJ, 494, L131

Menten, K. M., Eckart, A., Reid, M. J., \& Genzel, R. 1997, ApJ, 475, L111

Misner, C. W., Thorne, K. S., \& Wheeler, J. A. 1973 Gravitation (W. H. Freeman and Company, San Francisco)

Morris, M. 1993, ApJ, 408, 496

Munyaneza, F., Tsiklauri, D., \& Viollier, R. D. 1998, ApJ, 509, L105

Murphy, B. W., Cohn, H. N., \& Durisen, R. H. 1991, ApJ, 370, 60

Myoshi, M., Moran, J. M., Hernstein, J., et al. 1995, Nature, 373,127

Quirrenbach, A. 2000, From Extrasolar Planets to Cosmology: The VLT Opening Symposium, Proc. of an ESO Symposium held at Antofagasta, Chile, 1-4 March 1999, ed. J. Bergeron, \& A. Renzini (Berlin: Springer-Verlag, 2000), 462

Reid, M. 1993, ARA\&A, 31, 345

Reid, M. J., Readhead, A. C. S., Vermeulen, R. C., \& Treuhaft, R. N. 1999, ApJ, 524, 816

Richstone, D., et al. 1998, Nature, 395, 14

Saha, P., Bicknell, G. V., \& McGregor, P. J. 1996, ApJ, 467, 636

Schneider, M., Himmelsmechanik, Band III: 1996 Gravitationstheorie (Spektrum Akad. Verlag, Heidelberg)

Sellgren, K., McGinn, M. T., Becklin, E. E., \& Hall, D. N. B. 1990, ApJ, 359, 112

Swanson, P., Colavita, M., Boden, A., van Belle, G., \& Shao, M. 1997, AAS Meeting, 191, \#09.12

Taylor, J. H. 1993, Class. Quantum. Grav., 10, S167

von der Lühe, O., Derie, F., Koehler, B., et al. 1997, Proc. SPIE, 2871, 498

Wardle, M., \& Yusef-Zadeh, F. 1992, ApJ, 387, L65

Weinberg, S. 1972, Gravitation and Cosmology (Wiley, New York)

Will, C., 1993, Theory and experiment in gravitational physics, Revised Edition (Cambridge Univ. Press, Cambridge) 